FEDERAL

RESERVE

BANK of

ST. LOUIS
RESEARCH DIVISION

Working Paper Series

\title{
Optimal Disclosure Policy and Undue Diligence
}

\author{
David Andolfatto, \\ Aleksander Berentsen \\ and \\ Christopher J. Waller
}

\author{
Working Paper 2012-001A \\ https://doi.org/10.20955/wp.2012.001
}

December 2011

\author{
FEDERAL RESERVE BANK OF ST. LOUIS \\ Research Division \\ P.O. Box 442 \\ St. Louis, MO 63166
}

The views expressed are those of the individual authors and do not necessarily reflect official positions of the Federal Reserve Bank of St. Louis, the Federal Reserve System, or the Board of Governors.

Federal Reserve Bank of St. Louis Working Papers are preliminary materials circulated to stimulate discussion and critical comment. References in publications to Federal Reserve Bank of St. Louis Working Papers (other than an acknowledgment that the writer has had access to unpublished material) should be cleared with the author or authors. 


\title{
Optimal Disclosure Policy and Undue DiligenCE*
}

\author{
David Andolfatto \\ Federal Reserve Bank of St. Louis and Simon Fraser University \\ Aleksander Berentsen \\ University of Basel and Federal Reserve Bank of St. Louis \\ Christopher Waller \\ Federal Reserve Bank of St. Louis and University of Notre Dame \\ November 03, 2011
}

\begin{abstract}
While both public and private financial agencies supply asset markets with large amounts of information, they do not generally disclose all assetrelated information to the general public. This observation leads us to ask what principles might govern the optimal disclosure policy for an asset manager or financial regulator. To investigate this question, we study the properties of a dynamic economy endowed with a risky asset, and with individuals that lack commitment. Information relating to future asset returns is available to society at zero cost. Legislation dictates whether this information is to be made public or not. Given the properties of our environment, nondisclosure is generally desirable. This result is overturned, however, when individuals are able to access hidden information-what we call undue diligence-at sufficiently low cost. Information disclosure is desirable, in other words, only to the extent that individuals can easily discover it for themselves.
\end{abstract}

\footnotetext{
*The views expressed are those of the individual authors and do not necessarily reflect official positions of the Federal Reserve Bank of St. Louis, the Federal Reserve System, or the Board of Governors. Andolfatto: Federal Reserve Bank of St.Louis, P.O. Box 442 St. Louis, MO 63166-0442 (email David.Andolfatto@stls.frb.org). Berentsen: Economics Department, University of Basel, Peter-Merian-Weg 6, Postfach, CH-4002 Basel, Switzerland (email aleksander.berentsen@unibas.ch). Waller: Federal Reserve Bank of St.Louis, P.O. Box 442 St. Louis, MO 63166-0442 (email cwaller@stls.frb.org). For comments on earlier versions of this paper we thank Stephen Cecchetti, Leonardo Gambacorta, Jacob Gyntelberg, David Levine, Georg Noeldecke, Guillaume Rocheteau, Christian Upper, Randall Wright and participants in seminars or conferences at Banque de France, Bank for International Settlements, Federal Reserve Bank of St. Louis, Federal Reserve Bank of Chicago, Mannheim, St. Gallen, IHS Vienna, the Society for Economic Dynamics. Andolfatto acknowledges the financial support of SSHRC. Berentsen acknowledges support by the Bank for International Settlements (the usual disclaimer applies).
} 


\section{Introduction}

Financial agencies generally do not publicly disclose all information relating to the assets under their control or supervision. The phenomenon is evident in both the private and public sectors. Financial intermediaries, for example, are inclined not to report the current market value of their assets. ${ }^{1}$ Bank regulators do not publicly disclose their assessments of the individual bank balance sheets under their supervision. ${ }^{2}$ Central banks do not reveal the identity of those agencies making use of their emergency lending facilities. Many more examples exist, both presently and throughout history.

It is of some interest to note that nondisclosure policies are invariably defended by their practitioners on the grounds of promoting economic efficiency. While this idea may sound counterintuitive, there is a theoretical justification for the claim. In particular, since Hirshleifer (1971), economists have known that it is possible for information to possess negative social value, even if it has positive private value. In such a circumstance, individuals may collectively choose to design (or support) institutions that suppress the publication of socially harmful information. We will provide an example of just such an economy below.

On the other hand, attempts to encourage information disclosure in financial markets are also defended on the grounds of promoting economic efficiency. A prominent example of this is FASB Rule 157, which is legislation introduced in 2006 designed to encourage the use of "mark-to-market" valuation methods in the U.S. financial sector. Recent U.S. Congressional attempts to amend the U.S. Federal Reserve Act in a way that leaves the central bank with less discretion over its information disclosure policies may be another such example.

In reality, there are likely innumerable factors determining how information flows are governed in a society; and, in particular, whether or not some types of information are deemed (either voluntarily or by legislation) to be suitable for public viewing or not. The factor we want to focus on here is the technology of information acquisition itself. We suspect that the nature of this technology (or more broadly, any event that alters the cost-benefit calculation for private information acquisition) is likely to influence not only the pattern of economic activity, but perhaps even the socially desirable disclosure policy.

To investigate this question, we study a dynamic economy in which individuals lack commitment. A risky asset is used to facilitate intertemporal trade (expand the set of incentive-feasible allocations). Information that forecasts future asset returns is potentially available at zero cost; and society must choose whether to make this information available for public viewing or not. In the set-up considered here, it is generally desirable to suppress this particular infor-

\footnotetext{
${ }^{1}$ The tendency, instead, is to report values estimated from proprietary mark-to-model algorithms.

${ }^{2}$ Federal regulators, like the Fed, the OCC, and the FDIC do not disclose their so-called CAMELS ratings of private banks under supervision, for example.
} 
mation flow (or to disclose it with delay).

Nondisclosure is complicated, however, by the fact that individuals may discover hidden information on their own. Formally, we assume that individuals possess an information acquisition technology, similar to the "costly-stateverification" technology modeled in Townsend (1979). That is, individuals may, at some cost, surreptitiously discover on their own, what society wishes to remain hidden. We refer to this action as "undue diligence," because the activity, as we model it here, is harmful from the perspective of our social welfare criterion. ${ }^{3}$

We characterize sets of incentive-feasible allocations for different parameters, and under different disclosure policies. The threat of undue diligence generally restricts the set of incentive-feasible allocations, and can influence the socially optimal disclosure policy. ${ }^{4}$ Evidently, there are circumstances in which it is socially desirable to disclose harmful information; keeping it suppressed only makes matters worse. Almost paradoxically, information disclosure is optimal only in the event that individuals can easily discover it for themselves.

While a financial market is implicit in our "mechanism design" approach, it is easy to see how the model's properties can be mapped into decentralized solutions. The asset (or claims to the asset) in equilibrium circulate as a payment instrument or-equivalently-serve as collateral for a short-term loan. ${ }^{5}$ Information that leads to a downward revision in the forecast of future returns ("bad news") results in a decline in the asset's price and generally tightens debt constraints. These "credit crunch" events can be avoided here by suppressing news; which is tantamount to creating a type of "opacity" over the properties of an asset that plays an important role in the payments system. But if people can easily learn this news for themselves, it is better to let them have it for free, and suffer the consequences of bad news events when they occur.

Finally, we use our framework to interpret different views over what triggered the financial crisis of 2008. We discuss three hypotheses. The first hypothesis is that the introduction of a new financial product in 2006 essentially lowered the cost of information relating to subprime mortgage bonds. This event, together with the "unexpected" decline in real estate prices beginning in 2006, rendered a set of important debt instruments suddenly illiquid; see Gorton (2009a, 2009b). The second hypothesis is that the imposition of FASB 157 in 2007 forced banks to make transparent the (accounting) losses they were experiencing on their asset portfolios (owing to declining real estate prices), leading to a "firesale" dynamic that resulted in an "excessive" decline in asset prices. Finally, we

\footnotetext{
${ }^{3}$ That is, if people could commit beforehand not to partake in the activity of learning this information, they would do so.

${ }^{4}$ It turns out that constrained-efficient allocations are such that due diligence never actually occurs. That is, any allocation that induces due diligence can be replicated at lower cost simply by releasing sought-after information for free.

${ }^{5}$ Lagos and Rocheteau (2008) assume that capital is used as money; Ferraris and Watanabe (2008) assume instead that it is used as collateral for a money loan. The two interpretations are equivalent in these settings, as it is here.
} 
investigate how an increase in macroeconomic risk might in itself contribute to generating "credit crisis" phenomena.

\section{The Environment}

Our framework is based on the quasilinear model developed by Lagos and Wright [11]. Time is discrete and the horizon is infinite. Each time period is divided into two subperiods, labeled day and night.

The economy is populated by two types of agents, labeled $b$ and $s$ and referred to as buyers and sellers, respectively. Agents are infinitely-lived and there is an equal measure (a continuum) of each agent type. Agent types are defined by their characteristics at night. Let $q \in \mathbb{R}_{+}$denote output at night. Type $b$ agents want to consume this output, while type $s$ agents have an ability to produce it. Let $u(q)$ denote the utility associated with consumption and $-h(q)$ the utility associated with production. We apply the standard restrictions; $u^{\prime \prime}<0<u^{\prime}$ and $h^{\prime}, h^{\prime \prime}>0$. In addition, assume $u(0)=h(0)=0, u^{\prime}(0)=\infty$ and $h^{\prime}(0)=0$. There is a unique $0<q^{*}<\infty$ satisfying $u^{\prime}\left(q^{*}\right)=h^{\prime}\left(q^{*}\right)$.

Agents have linear utility $y \in \mathbb{R}$ in the day (utility is transferable, with negative values interpreted as production), and agents have a common discount factor $0<\beta<1$. Ex ante utility functions, for type $b$ and $s$ agents respectively, are given by:

$$
\begin{aligned}
& W_{b}=E_{0} \sum_{t=0}^{\infty} \beta^{t}\left[y_{b, t}+u\left(q_{b, t}\right)\right] \\
& W_{s}=E_{0} \sum_{t=0}^{\infty} \beta^{t}\left[y_{s, t}-h\left(q_{s, t}\right)\right]
\end{aligned}
$$

There is a single asset in the economy; a "Lucas tree." The asset generates a stochastic flow of nondurable output (utility) $z_{t} \geq 0$ at the beginning of each day. For simplicity, this dividend is assumed to take on two values, $0 \leq z^{l}<$ $z^{h}<\infty$. Let $\pi \equiv \operatorname{Pr}\left[z_{t}=z^{h}\right]$ denote the probability of a high dividend, and let $\bar{z} \equiv \pi z^{h}+(1-\pi) z^{l}$.

\subsection{Information}

Society is in possession of a machine that generates information at zero cost. The information generated by this machine arrives at night and reveals the next day's dividend. ${ }^{6}$

The information machine may be programmed to communicate (or withhold) information to (from) all members of society. Think of the machine as possessing

\footnotetext{
${ }^{6}$ It is straightforward to show that our arguments hold even if the information is not perfectly correlated with the dividend realized the next day.
} 
an on/off communication switch. The switch position is chosen by society (or a mechanism with the goal of maximizing a given social welfare function). If society turns the switch on, the one-period-ahead dividend is communicated to the public. We refer to this case as full disclosure. If society turns the switch off, this information is not communicated to the public. We refer to this case as nondisclosure. Under nondisclosure, a deliberate vagueness is attached to the asset's underlying fundamentals.

The main purpose of our analysis below is to discover under what conditions society will find it desirable to turn the communication switch on or off. Unless otherwise stated, we assume full record-keeping.

\section{$2.2 \quad$ Allocations}

In everything that follows, we restrict attention to symmetric stationary allocations, denoted by the list $\left(q^{l}, q^{h}, y_{s}^{l}, y_{s}^{h}, y_{b}^{l}, y_{b}^{h}\right)$.

An allocation must satisfy resource feasibility in the day; i.e.,

$$
y_{s}^{i}+y_{b}^{i}=z^{i},
$$

for $i=l, h$. Let $\bar{y}_{s} \equiv \pi y_{s}^{h}+(1-\pi) y_{s}^{l}$ and $\bar{y}_{b} \equiv \pi y_{b}^{h}+(1-\pi) y_{b}^{l}$. Note that these latter two resource constraints imply

$$
\bar{y}_{s}+\bar{y}_{b}=\bar{z} .
$$

An allocation must also respect resource feasibility at night. Feasibility requires

$$
q_{b}^{i}=q_{s}^{i}=q^{i} \geq 0
$$

for $i=l, h$.

A feasible allocation satisfies (3) and (5). Using (3), we economize on notation and denote a feasible allocation by $\left(q^{l}, q^{h}, y_{s}^{l}, y_{s}^{h}\right)$.

We make note of two things that should be kept in mind for what follows. First, if the communication switch is turned off, a feasible allocation is further restricted to satisfy $q^{l}=q^{h}$; that is, the night allocation must be made nonstate-contingent (since information would otherwise be communicated indirectly by the proposed allocation). Second, if the communication switch is turned on, the night allocation need not be made state-contingent; that is, $q^{l}=q^{h}$ remains a possibility.

\subsection{Efficiency under commitment}

In the following analysis, we speak of a "mechanism" that proposes (or recommends) an allocation $\left(q^{l}, q^{h}, y_{s}^{l}, y_{s}^{h}\right)$ that people are free to accept or reject. ${ }^{7}$

\footnotetext{
${ }^{7}$ This is somewhat weaker than the conventional notion of a "planner" who has the power to command the allocation of resources.
} 
As a benchmark, we consider the allocation that maximizes the sum of ex ante utility payoffs (1) and (2) when individuals have full commitment. While it is feasible to condition the night allocation on information, in this environment it is clearly not desirable to do so. That is, any efficient allocation will have the property $q^{i}=q^{*}$ for $i=l, h$. An efficient allocation delivers ex ante welfare,

$$
\begin{aligned}
& (1-\beta) W_{b}=\bar{y}_{b}+u\left(q^{*}\right), \\
& (1-\beta) W_{s}=\bar{y}_{s}-h\left(q^{*}\right),
\end{aligned}
$$

with $\left(\bar{y}_{b}, \bar{y}_{s}\right)$ divided in any manner that satisfies (4) and does not violate the ex ante participation constraints $W_{s}, W_{b} \geq 0$. The fact that $q^{*}$ is insensitive to information demonstrates that the information flow at night possesses zero social value in this environment (this is by construction). Under full commitment, disclosure policy is irrelevant.

In what follows, we assume that individuals lack commitment and that noncompliance (the rejection of a recommended allocation) is punished with ostracism. We investigate two cases in turn-one in which the communication switch is turned on, and one in which it is turned off. Proofs for all propositions are relegated to an appendix.

\section{Full disclosure}

In this section, we turn the communication switch on and characterize the set of incentive-feasible allocations (feasible allocations that respect a set of sequential participation constraints). In particular, we want to determine the conditions under which the efficient allocation is incentive feasible.

When the communication switch is on, the recommended allocation at night can be conditioned on the future (next day) dividend state. Because sellers incur sacrifice (production) at night, and buyers incur sacrifice in the day, there are two relevant SP constraints to consider.

In the case considered here, the seller is asked to produce $q^{l}$ for the future reward $y_{s}^{l}$, or $q^{h}$ for the future reward $y_{s}^{h}$. (We remind the reader that $q^{l}=q^{h}$ is a possibility here.) The sequential participation (SP) constraints for the seller at night are given by

$$
-h\left(q^{i}\right)+\beta\left(y_{s}^{i}+V_{s}\right) \geq \beta^{T} V_{s}, i=l, h,
$$

where $V_{s} \equiv(1-\beta)^{-1}\left(-\bar{h}+\beta \bar{y}_{s}\right)$ is the continuation value for a seller who follows a recommended allocation with $\bar{h} \equiv \pi h\left(q^{h}\right)+(1-\pi) h\left(q^{l}\right)$. The parameter $T$ governs the severity of the punishment associated with noncompliance. Setting $T=\infty$, for example, implies perpetual ostracism.

The set of SP constraints for the buyer in the day are given by

$$
y_{b}^{i}+\bar{u}+\beta W_{b} \geq \beta^{T} W_{b} \text { for } i \in\{l, h\},
$$


where $W_{b} \equiv(1-\beta)^{-1}\left(\bar{y}_{b}+\bar{u}\right)$ with $\bar{u} \equiv \pi u\left(q^{h}\right)+(1-\pi) u\left(q^{l}\right)$. Here, $W_{b}$ is the continuation value for a buyer who follows a recommended allocation. Note that $\bar{u}$ reflects the fact that the buyer does not yet know the state that will prevail at night.

Lemma 1 Consider any $\left(q^{l}, q^{h}, y_{b}^{l}, y_{b}^{h}\right)$ that satisfies (7), then $\left(q^{l}, q^{h}, \bar{y}_{b}, \bar{y}_{b}\right)$ necessarily satisfies (7).

Lemma 1 follows from the fact that $\bar{y}_{b} \geq \min \left(y_{b}^{l}, y_{b}^{h}\right)$, so that one may, without loss of generality, restrict attention to incentive-feasible allocations that satisfy $y_{b}^{l}=y_{b}^{h}=\bar{y}_{b}$. For $y_{b}^{l}=y_{b}^{h}=\bar{y}_{b}$, the SP constraint of the buyer is

$$
\bar{y}_{b}+\bar{u}+\beta W_{b} \geq \beta^{T} W_{b},
$$

This latter expression implies the following SP constraint of the buyer: ${ }^{8}$

$$
\bar{y}_{s} \leq \bar{z}+\bar{u}
$$

Definition 1 When the communication switch is on, an incentive-feasible allocation is a feasible allocation $\left(q^{l}, q^{h}, y_{s}^{l}, y_{s}^{h}\right)$ that satisfies (6) and (9).

Proposition 1 If the communication switch is on, an incentive-feasible allocation exists for any $\left(q^{l}, q^{h}\right) \in S^{l} \cap S^{h}$, a non-empty and compact set, where

$$
\begin{aligned}
S^{l} & \equiv\left\{\left(q^{l}, q^{h}\right) \in \mathbb{R}_{+}^{2}:-h\left(q^{l}\right)+\beta\left(\bar{u}+z^{l}\right)-\left(\beta^{T}-\beta\right) V_{s} \geq 0\right\}, \\
S^{h} & \equiv\left\{\left(q^{l}, q^{h}\right) \in \mathbb{R}_{+}^{2}:-h\left(q^{h}\right)+\beta\left(\bar{u}+z^{h}\right)-\left(\beta^{T}-\beta\right) V_{s} \geq 0\right\} .
\end{aligned}
$$

Whether the efficient night-output $\left(q^{*}, q^{*}\right)$ is an element of the $S^{l} \cap S^{h}$ evidently depends on the parameters. We define the following objects:

$$
\begin{aligned}
\Omega & \equiv(1-\pi)\left(1-\beta^{T}\right)(1-\beta)^{-1} \geq 1 \\
\bar{\gamma} & \equiv \pi(1-\pi) \beta\left(z^{h}-z^{l}\right) \\
\bar{z}^{N} & \equiv \beta^{-1}\left[h\left(q^{*}\right)-\beta u\left(q^{*}\right)\right] .
\end{aligned}
$$

Proposition 2 If the communication switch is turned on, the efficient night allocation $\left(q^{*}, q^{*}\right)$ is incentive feasible for all $\bar{z} \geq \bar{z}^{F}$, where $\bar{z}^{F}$ satisfies

$$
\bar{z}^{F} \equiv \bar{z}^{N}+\frac{\bar{\gamma}}{\Omega \beta}
$$

\footnotetext{
${ }^{8}$ To see this, replace $W_{b} \equiv(1-\beta)^{-1}\left(\bar{y}_{b}+\bar{u}\right)$ in $(8)$ to get $(1-\beta)^{-1}\left(1-\beta^{T}\right)\left(\bar{y}_{b}+\bar{u}\right) \geq 0$. Since $(1-\beta)^{-1}\left(1-\beta^{T}\right)>0$, we get $\bar{y}_{b}+\bar{u} \geq 0$. Finally, use (4) to replace $\bar{y}_{b}=\bar{z}-\bar{y}_{s}$ to get $(9)$.
} 
When the asset's return is sufficiently high, sellers are willing to produce $q^{*}$. If $\bar{z}<\bar{z}^{F}$, the efficient night allocation $\left(q^{*}, q^{*}\right)$ is not incentive feasible. In this case, it may be constrained-efficient to recommend a state-contingent allocation. When this is the case, the mechanism is willing to trade off consumption volatility for a cheaper way to induce sequential participation. An example of such a constrained-efficient night output is illustrated as the intersection point of two curves (high and low) in Figure 1.

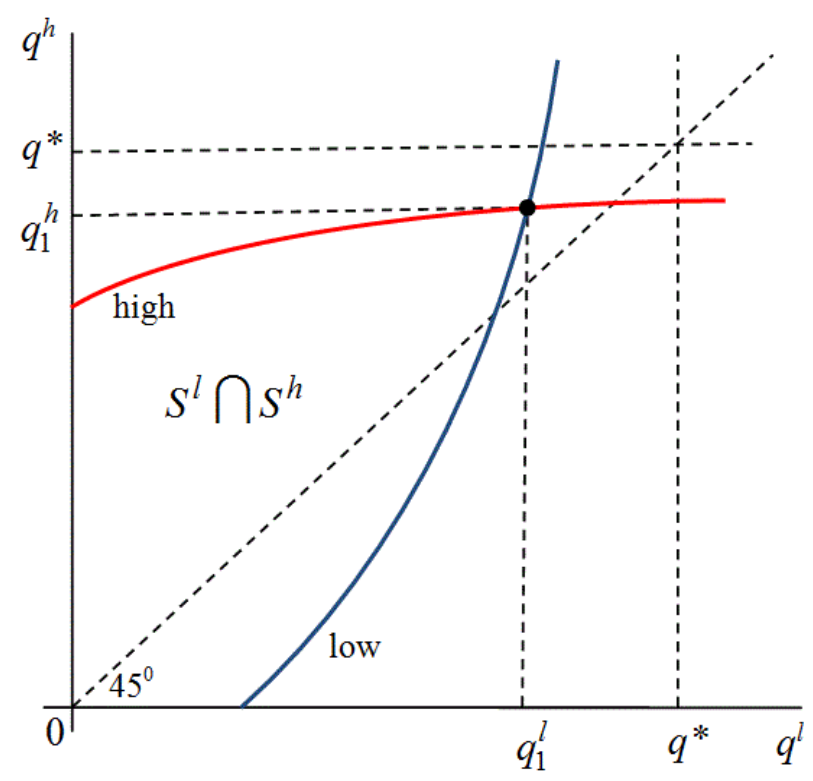

Figure 1: Incentive-feasible quantities $\left(q^{l}, q^{h}\right)$.

In Figure 1, the curve labeled low (high) represents combinations of $q^{l}$ and $q^{h}$ such that SP constraint (10) [(11)] just holds. The constrained-efficient level of production at night is $\left(q_{1}^{l}, q_{1}^{h}\right)$ with $q_{1}^{l}<q_{1}^{h}$. That is, to induce participation in the low state, the seller is asked to produce less than $q_{1}^{h}$ (there is a shortage of future utility to finance rewards in the low state).

The incentive-feasible set $S^{l} \cap S^{h}$ here is derived under the assumption of full information disclosure. The question we ask next is how nondisclosure affects incentive-feasibility.

\section{Nondisclosure}

In this section, we turn the communication switch off and characterize the set of incentive-feasible allocations. Again, we want to determine conditions under which the efficient allocation is incentive feasible. 
Recall that if the communication switch is turned off, a feasible allocation $\left(q^{l}, q^{h}, y_{s}^{l}, y_{s}^{h}\right)$ is restricted to satisfy $q^{l}=q^{h}=q$. That is, the seller is asked to produce $q$ in each state and, since the state is not communicated, the seller's future reward is the expected payoff $\bar{y}_{s}$.

The SP constraints for a seller at night are given by:

$$
-h(q)+\beta\left(\bar{y}_{s}+V_{s}\right) \geq \beta^{T} V_{s},
$$

where $V_{s} \equiv(1-\beta)^{-1}\left[-h(q)+\beta \bar{y}_{s}\right]$ is the continuation value for a seller who follows the recommended allocation. This latter expression implies the following SP constraint for the seller: ${ }^{9}$

$$
-h(q)+\beta \bar{y}_{s} \geq 0 .
$$

Let $S \equiv\left\{\left(q, \bar{y}_{s}\right):-h(q)+\beta \bar{y}_{s} \geq 0\right\}$ denote the set of allocations $\left(q, \bar{y}_{s}\right)$ that induce SP for the seller. Given the assumed properties for $h$, the set $S$ is clearly non-empty and strictly convex.

The SP constraints for the buyer in the day are given by

$$
y_{b}^{i}+u(q)+\beta W_{b} \geq \beta^{T} W_{b}, i \in\{l, h\},
$$

where $W_{b} \equiv(1-\beta)^{-1}\left[\bar{y}_{b}+u(q)\right]$. Here, $W_{b}$ is the continuation value for a buyer who follows the recommended allocation. The buyer is asked to produce $y_{b}^{i}$ in states $i=l, h$ and his period reward is the payoff $u(q)$.

Using Lemma 1 and arguments similar to those used in deriving (9), one can show that if an allocation satisfies the SP constraint for the buyer (18), it also satisfies the inequality

$$
\bar{y}_{s} \leq \bar{z}+u(q),
$$

which conveniently allows us to express incentive-feasible allocations for the buyer in terms of $\left(q, \bar{y}_{s}\right)$. Let $B \equiv\left\{\left(q, \bar{y}_{s}\right): \bar{y}_{s} \leq \bar{z}+u(q)\right\}$ denote the set of allocations $\left(q, \bar{y}_{s}\right)$ that satisfy SP for the buyer. Given the assumed properties of $u$, the set $B$ is clearly non-empty and convex.

Definition 2 When the communication switch is off, an incentive-feasible allocation is a feasible allocation $\left(q^{l}, q^{h}, y_{s}^{l}, y_{s}^{h}\right)$ with $q^{l}=q^{h}$ that satisfies (17) and (19).

If the communication switch is turned off, the set of incentive-feasible allocations is given by $S \cap B$; a non-empty and compact set.

Proposition 3 If the communication switch is off, there exists an incentivefeasible allocation for any $q \in\left[0, q_{m}\right]$, where $q_{m} \geq 0$ solves

$$
\beta\left[\bar{z}+u\left(q_{m}\right)\right]-h\left(q_{m}\right) \equiv 0 .
$$

\footnotetext{
${ }^{9}$ To see this, replace $V_{s} \equiv(1-\beta)^{-1}\left[-h(q)+\bar{y}_{s}\right]$ in (16) to get $(1-\beta)^{-1}(1-$ $\left.\beta^{T}\right)\left[-h(q)+\bar{y}_{s}\right] \geq 0$. Since $(1-\beta)^{-1}\left(1-\beta^{T}\right)>0$, we get $(17)$.
} 
The quantity $q_{m}$ is the largest value of $q$ such that the SP constraints of buyers and sellers hold. ${ }^{10}$ Whether $q^{*} \in\left[0, q_{m}\right]$ depends on parameters. If $q^{*} \leq q_{m}$, the efficient quantity is incentive feasible.

Proposition 4 If the communication switch is off, the efficient night allocation $\left(q^{*}, q^{*}\right)$ is incentive feasible for all $\bar{z} \geq \bar{z}^{N}$, where

$$
\bar{z}^{N} \equiv \beta^{-1}\left[h\left(q^{*}\right)-\beta u\left(q^{*}\right)\right]<\bar{z}^{F} .
$$

Propositions 2 and 4 immediately imply that the efficient allocation can be supported over a larger range of values for $\bar{z}$ under nondisclosure, since $\bar{z}^{F}>\bar{z}^{N}$.

We have to this point studied two cases. The mechanism was designed to communicate information or not. In the latter case, it was implicitly assumed that individuals had no ability to acquire this information on their own. In what follows, we extend the model by endowing individuals with a costly informationacquisition technology.

Naturally, under a policy of full disclosure, there will be no individual incentive to acquire information that is freely available. For this reason, in the following section we continue to assume that the mechanism does not disclose information.

\section{Undue diligence}

Assume now that individuals have a technology that allows them, at night and at utility cost $\gamma \geq 0$, to learn the next-day dividend state.

In what follows, we depart from our assumption of full record-keeping. That is, while individual consumption and production histories remain publicly observable, actions relating to information acquisition are not. Thus, whether an agent chooses to operate the information acquisition technology, together with what he learns from its operation, remains private information. ${ }^{11}$

In this environment, information acquisition constitutes a social waste. The information has no social benefit and, in addition, there is an acquisition cost.

\footnotetext{
${ }^{10}$ Note that at $q=q_{m}$ both SP constraints bind, so that $V_{s}=W_{b}=0$. Moreover, $W_{s}=$ $\beta^{-1} h\left(q_{m}\right)$ and $V_{b}=u\left(q_{m}\right)$. From (1) and (2) the period surplus is $(1-\beta)\left(W_{s}+W_{b}\right)=$ $\bar{z}+u\left(q_{m}\right)-h\left(q_{m}\right)$. Using the previous expressions to replace $W_{s}$, we get $(1-\beta) W_{s}=\bar{z}+$ $u\left(q_{m}\right)-h\left(q_{m}\right)$. That is, the seller receives the entire ex ante surplus.

${ }^{11}$ The reason for this restriction on public record-keeping is simple. If information gathering activies are observable, then such activities can be punished as with any act of noncompliance. The possibility of information gathering would then be irrelevant in the sense that it does not restrict the set of incentive-feasible allocations.

We also assume that information gathered in this manner cannot be communicated to others. This is without loss of generality, since if an agent communicates the state, he reveals that he has used the information acquisition technology which then could be punished by the mechanism.
} 
Nevertheless, there may exist private incentives to acquire information. Moreover, its acquisition may inhibit ex ante efficient trades from occurring. What is the intuition for this?

Consider an incentive-feasible allocation with $y_{s}^{l}<y_{s}^{h}$. (Note that when $\bar{z}=\bar{z}^{N}$, the efficient allocation is incentive feasible and necessarily has this latter property.) For any such allocation, the seller is asked to produce $q$ at night, essentially, in exchange for promised (expected) utility $\bar{y}_{s}$ the next day. One property of this allocation is that, ex post, the seller faces a lower reward in the low-dividend state. Consequently, it is conceivable that the seller might not want to produce at level $q$ if he knew beforehand that the asset will have a low yield. If the cost of obtaining this information is sufficiently low, then he will have an incentive to acquire it for the purpose of rejecting the trade in the event of "bad news." 12

The incentive to acquire information is absent if and only if the following condition holds,

$$
-h(q)+\beta\left(\bar{y}_{s}+V_{s}\right) \geq-\gamma+\pi\left[-h(q)+\beta\left(y_{s}^{h}+V_{s}\right)\right]+(1-\pi) \beta^{T} V_{s} .
$$

The left-hand side of (20) denotes the utility payoff from accepting the recommended allocation without bothering to gather information. The right-hand side of (20) denotes the utility payoff associated with information acquisition. Gathering information entails the direct utility cost $\gamma$ to the seller. The recommendation is accepted only in the event of "good news." (It can never be optimal to gather costly information and then not condition future behavior on it.) Rejecting the recommendation yields the continuation payoff $\beta^{T} V_{s}$.

Using $V_{s} \equiv(1-\beta)^{-1}\left[-h(q)+\beta \bar{y}_{s}\right]$ to replace $V_{s}$ in $(20)$ and rearranging, the condition that deters information acquisition can be written as

$$
\gamma \geq(1-\pi)\left[h(q)-\beta y_{s}^{l}\right]-(1-\pi)(1-\beta)^{-1}\left(\beta-\beta^{T}\right)\left[\beta \bar{y}_{s}-h(q)\right] .
$$

The first term on the right-hand side of (21) is the seller's gain from not producing in the low state. The second term on the right-hand side of (21) is the seller's cost of being ostracized for $T$ periods. Thus, the smaller is the gain of not producing in the low state $\left[h(q)-\beta y_{s}^{l}\right]$ and the greater is the expected period surplus accruing to the seller $\left[\beta \bar{y}_{s}-h(q)\right]$, the easier it will be to deter the gathering of information. The terms are multiplied by the probability of a low state-the only state in which the seller does not produce.

We want to stress here that (21) is not to be viewed as a constraint; rather, it is simply a condition that must be satisfied if sellers are to be deterred from acquiring their own information. As we shall see later on, it may be in the social interest not to have this condition hold.

\footnotetext{
${ }^{12}$ Note that the buyer has no incentive to acquire information, because his participation choice occurs when the state is known.
} 
Moving along, note that one can rewrite (21) along the same lines as the SP constraint of the seller (17) to get ${ }^{13}$

$$
\bar{y}_{s} \geq \beta^{-1} h(q)+(\beta \Omega)^{-1}(\bar{\gamma}-\gamma) .
$$

where $\Omega$ is defined in (12). Let $N \equiv\left\{\left(q, \bar{y}_{s}\right): \bar{y}_{s}-\beta^{-1} h(q)-(\beta \Omega)^{-1}(\bar{\gamma}-\gamma) \geq 0\right\}$ denote the set of allocations $\left(q, \bar{y}_{s}\right)$ that induce no information gathering by the seller. Given the assumed properties for $h$, the set $N$ is clearly non-empty and strictly convex.

Definition 3 When the communication switch is off, an incentive-feasible allocation that does not promote inspection by sellers is a feasible allocation $\left(q, \bar{y}_{s}\right)$ that satisfies (17), (19), and (22).

To recap, when the communication switch is turned off, the set of incentivefeasible allocations is given by $S \cap B$. Clearly, the set of incentive-feasible allocations that does not promote information-gathering must be a subset of $S \cap B$, since we added condition (22). The question is whether it is a strict subset. The answer turns out to be yes if the inspection cost $\gamma$ is sufficiently low.

Proposition 5 If $\gamma<\bar{\gamma}$, then $N \subset S$ and $N \cap B \subset S \cap B$.

Figure 2 is a graphical representation of Proposition 5. The curve labeled $u(q)+\bar{z}$ represents combinations of $q$ and $\bar{y}_{s}$ such that the buyer's SP constraint is just binding. The curve labeled $\beta^{-1} h(q)+(\beta \Omega)^{-1}(\bar{\gamma}-\gamma)$ represents combinations of $q$ and $\bar{y}_{s}$ such that the seller is just indifferent between gathering information and not. Finally, the curve labeled $\beta^{-1} h(q)$ represents combinations of $q$ and $\bar{y}_{s}$ such that the seller's SP constraint is just binding. If $\gamma<\bar{\gamma}$, the condition that deters information acquisition restricts the set of incentivefeasible allocations. When this is so, the highest level of night-output that does not promote inspection by the seller is depicted in Figure 2 by the quantity $q_{0}<q_{m} \cdot{ }^{14}$

\footnotetext{
${ }^{13}$ As shown in Lemma 1 , we may set $y_{b}^{l}=y_{b}^{h}=\bar{y}_{b}$ without loss of generality. This, together with feasibility, implies that $y_{s}^{l}=z^{l}-\bar{y}_{b}=z^{l}-\left(\bar{z}-\bar{y}_{s}\right)$. Replacing $y_{s}^{l}$ in (21) and rearranging yields (22).

${ }^{14}$ Recall that $q_{m}$ is the largest quantity that satisfies the SP constraints of buyers and sellers when the communication switch is off. See Proposition 3.
} 


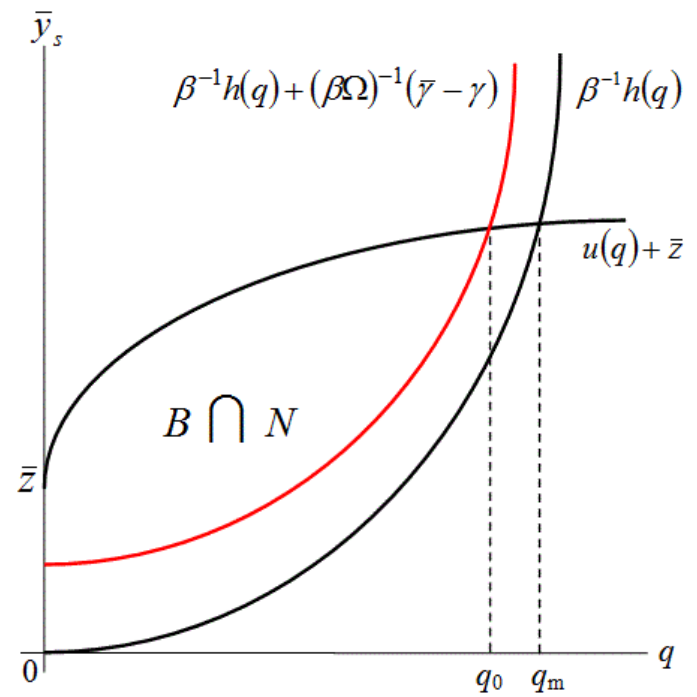

Figure 2: Incentive-feasible allocations when $\gamma<\bar{\gamma}$.

Proposition 6 If the communication switch is off, then for any $\gamma<\bar{\gamma}$, there exists an incentive-feasible allocation that does not promote information acquisition for any $q \in\left[0, q_{0}\right]$; where $q_{0}$ is the largest $q$ satisfying

$$
\bar{z}+u(q)-\beta^{-1} h(q)-(\beta \Omega)^{-1}(\bar{\gamma}-\gamma)=0 .
$$

Whether $q^{*}$ belongs to the set $\left[0, q_{0}\right]$ depends on parameters. If $q^{*} \leq q_{0}$, then the efficient quantity is incentive feasible and does not promote information acquisition by the seller.

Proposition 7 If the communication switch is off and if $\bar{z} \geq \max \left\{\bar{z}^{N}, \bar{z}^{D}\right\}$, the efficient night allocation $\left(q^{*}, q^{*}\right)$ is incentive feasible, where

$$
\bar{z}^{D} \equiv \bar{z}^{N}+\frac{\bar{\gamma}-\gamma}{\Omega \beta}
$$

The efficient allocation is incentive feasible if two conditions are met. First, the seller's SP constraint has to be satisfied $\left(\bar{z} \geq \bar{z}^{N}\right)$. Second, the "noinspection-condition" (22) has to be satisfied $\left(\bar{z} \geq \bar{z}^{D}\right)$. The latter condition depends negatively on $\gamma$. For $\gamma<\bar{\gamma}$, the no-inspection-condition is the binding constraint, since $\bar{z}^{D}>\bar{z}^{N}$. For $\gamma>\bar{\gamma}$, the seller's SP constraint is the binding constraint, since $\bar{z}^{D}<\bar{z}^{N}$. 


\section{Optimal communication policy}

In this section, we derive the optimal communication policy. To this end, we need to compare the welfare properties of the incentive-feasible allocations when the communication switch is off and when it is on. For any allocation, our welfare criterion is

$$
(1-\beta) W\left(q^{l}, q^{h}\right) \equiv \bar{u}\left(q^{l}, q^{h}\right)-\bar{h}\left(q^{l}, q^{h}\right)+\bar{z}
$$

To discuss the optimal communication policy, it is useful to consider Figure 3 , which divides the parameter space $(\gamma, \bar{z})$ into four regions, labeled A,B,C and D. Each region tells us the nature of the constrained-efficient allocation, as well as whether it is optimal to turn the communication switch on or off. We discuss the regions in alphabetic order below.

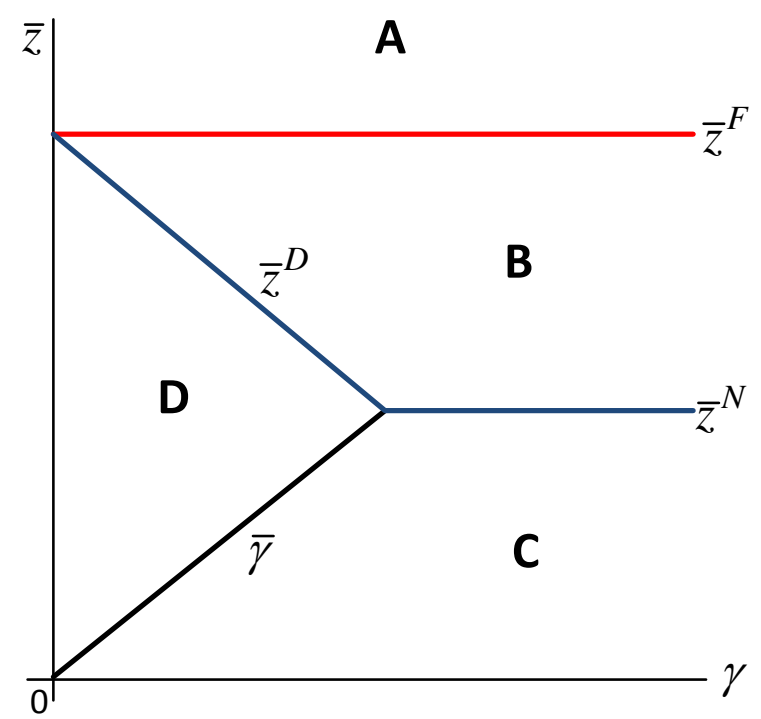

Figure 3. Optimal communication policies.

The red curve (labelled $\bar{z}^{F}$ ) in Figure 3 represents combinations of $\bar{z}$ and $\gamma$ such that $\bar{z}=\bar{z}^{F}$. The blue curve (labelled $\bar{z}^{N}$ and $\bar{z}^{D}$ ) represents combinations of $\bar{z}$ and $\gamma$ such that $\bar{z}=\max \left\{\bar{z}^{N}, \bar{z}^{D}\right\}$. The black curve (labelled $\bar{\gamma}$ ) represents combinations of $\bar{z}$ and $\gamma$ such that $\gamma=\bar{\gamma}{ }^{15}$

Region A Region A describes the set of economies where the expected return of the asset is high. The efficient allocation is incentive feasible when the

\footnotetext{
${ }^{15}$ This curve satisfies $\gamma=\beta(1-\pi) \pi\left(z^{h}-z^{l}\right)$. We can rewrite it in terms of $\bar{z}$ to get $\bar{z}=z^{l}+\frac{\gamma}{(1-\pi) \beta}$. Thus, the curve is increasing in $\gamma$ and has the intercept $z^{l}$ which we have set to zero in Figure 3.
} 
communication switch is on, since $\bar{z} \geq \bar{z}^{F}$ (see Proposition 2). Moreover, it is also incentive feasible when the switch is off, since $\bar{z}^{F} \geq \max \left\{\bar{z}^{N}, \bar{z}^{D}\right\}$ (see Proposition 7).

Thus, in region A, one may leave the communication switch on or off; disclosure policy is irrelevant.

Region B Region B describes a set of economies where a moderate expected return of the asset is combined with relatively high information acquisition costs. In this region, the efficient allocation is not incentive feasible when the communication switch is on, since $\bar{z}<\bar{z}^{F}$ (see Proposition 2). It is, however, incentive feasible when the switch is off, since $\bar{z} \geq \max \left\{\bar{z}^{N}, \bar{z}^{D}\right\}$ (see Proposition 7).

Thus, in region B, the first-best allocation is incentive feasible if (and only if) the communication switch off. Nondisclosure is part of the optimal policy here.

Region C Region C describes a set of economies where a low expected return of the asset is combined with relatively high information acquisition costs. In this region, the efficient allocation is not incentive feasible when the communication switch is on or off, since $\bar{z} \leq \max \left\{\bar{z}^{N}, \bar{z}^{D}\right\} \leq \bar{z}^{F}$. Furthermore, the no-inspection-condition (22) is nonbinding, since $\gamma \geq \bar{\gamma}$ (see Proposition 5).

To derive the optimal communication policy, we have to compare the welfare levels associated with the constrained-efficient allocations under full disclosure and nondisclosure, respectively. Note that if the switch is off, the welfare maximizing night output is $q_{m}$, since the no-inspection-condition is nonbinding. We have the following result:

Proposition 8 If $\bar{z}<\bar{z}^{N}$ and $\gamma \geq \bar{\gamma}$, then the optimal communication policy is to turn the switch off. The constrained-efficient allocation is given by

$$
\left(q, \bar{y}_{s}\right)=\left(q_{m}, \bar{z}+u\left(q_{m}\right)\right) .
$$

Consequently, if $\bar{z}<\bar{z}^{N}$ and if the no-inspection-condition is nonbinding, nondisclosure is part of the constrained-efficient policy.

Region D Region D describes a set of economies whose predominant characteristic is the availability of low-cost information acquisition technologies (these economies are also characterized by moderate to low patience). In this region, the efficient allocation is not incentive feasible when the communication switch is on or off, since $\bar{z} \leq \max \left\{\bar{z}^{N}, \bar{z}^{D}\right\} \leq \bar{z}^{F}$. Furthermore, the no-inspectioncondition is binding, since $\gamma<\bar{\gamma}$ (see Proposition 5). 
Proposition 9 If $\gamma \leq \bar{\gamma}$ and $\bar{z}<\bar{z}^{D}$, then there exists a critical value $0<\hat{\gamma}<\bar{\gamma}$ such that if $\bar{\gamma} \geq \gamma \geq \hat{\gamma}$, then it is optimal to turn the communication switch off, and if $0 \leq \gamma \leq \hat{\gamma}$, then it is optimal to turn the switch on.

Consequently, if $\gamma$ and $\bar{z}$ are sufficiently low, market participants are better off having information disclosed to them. While doing so introduces suboptimal variation in the night-allocation, it has the benefit of deterring undue diligence and allowing for a higher average level of night output.

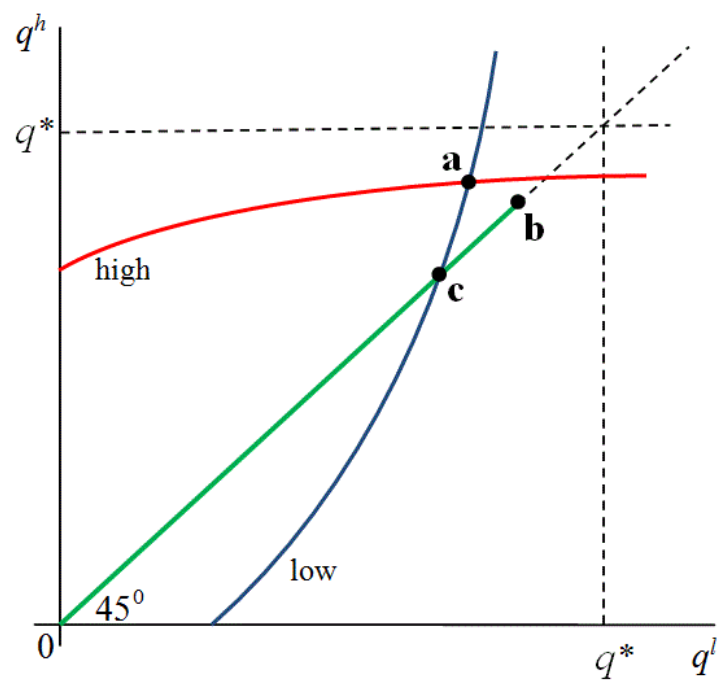

Figure 4. Proof of Proposition 9.

The results of Proposition 9 are now explained with the help of Figure 4. Note that Figure 4 is drawn for a given value of $\gamma$.

When the switch is on, any incentive-feasible night allocation $\left(q^{l}, q^{h}\right)$ belongs to the set $S^{l} \cap S^{h}$ (the region between the curves labeled high and low; see also Figure 1). In this case, the constrained-efficient night allocation is given by point a. Note that point $\mathbf{c}$ is on the intersection of the 45 degree line and the curve labeled low, where this latter curve represents combinations of $q^{l}$ and $q^{h}$ that make the seller's SP constraint just binding when the state is low. Evidently, allocation a yields a higher welfare level than allocation $\mathbf{c}$. Recall that when the switch is on, the value of $\gamma$ does not affect the set $S^{l} \cap S^{h}$, and hence it neither affects a nor $\mathbf{c}$.

From Proposition 6, when the switch is off and $\gamma<\bar{\gamma}$, the welfare maximizing night allocation is $\left(q_{0}, q_{0}\right)$, labeled point $\mathbf{b}$ in Figure 4. From Proposition 6 , allocation $\mathbf{b}$ is a function of $\gamma$ with $d q_{0} / d \gamma>0$. Thus, as we increase $\gamma$, point b moves up along the 45-degree line. That is, as we increase $\gamma$, the welfare maximizing level of night output is increasing (along with welfare). 
In the proof of Proposition 6, we evaluate the welfare level attained with $\mathbf{b}$ at $\gamma=0$ and at $\gamma=\bar{\gamma}$, and compare these welfare levels with the welfare level attained at $\mathbf{a}$. First, we show that at $\gamma=0$, allocations $\mathbf{b}$ and $\mathbf{c}$ coincide. Thus, since welfare at point $\mathbf{a}$ is strictly larger than welfare at point $\mathbf{c}$, we can immediately conclude that at $\gamma=0$, the optimal policy is to turn the switch on and to choose point a. Continuity then implies that for values of $\gamma$ that are sufficiently close to zero, the optimal policy is to turn the switch on and to choose point $\mathbf{a}$.

For larger values of $\gamma$, however, allocation $\mathbf{b}$ can yield a higher welfare level than allocation a. To see this, we have to distinguish two cases: $\bar{z} \geq \bar{z}^{N}$ and $\bar{z}<\bar{z}^{N}$. If $\bar{z} \geq \bar{z}^{N}$ and $\gamma=\bar{\gamma}$, we show that point $\mathbf{b}$ corresponds to the efficient night allocation $\left(q^{*}, q^{*}\right)$. Thus, in this case, it is clear that if $\gamma$ is close to $\bar{\gamma}$, the optimal policy is to turn the switch off and choose allocation b. If $\bar{z}<\bar{z}^{N}$ and $\gamma=\bar{\gamma}$, we show that point $\mathbf{b}$ corresponds to allocation $\left(q_{m}, q_{m}\right)$. For this case, we also show that the optimal policy is to turn the switch off and choose allocation $\mathbf{b}$. Continuity then implies that for values of $\gamma$ that are sufficiently close to $\bar{\gamma}$, the optimal policy is to turn the switch off, and to choose allocation b.

To recap the optimal communication strategy in region D: If $\gamma$ is sufficiently low, it is optimal to turn the communication switch on and to choose the statecontingent allocation a. If $\gamma$ is sufficiently close to $\bar{\gamma}$, it is optimal to turn the communication switch off and choose the non-state-contingent allocation $\mathbf{b}$.

Figure 4 is also useful for demonstrating how the set of incentive-feasible allocations $S^{l} \cap S^{h}$ collapses (to a segment of the $45^{0}$-line indicated in green) when information is made unavailable. This accords with the common-sense notion that withholding information imposes additional constraints. On the other hand, withholding information also has the effect of expanding the set of incentive-feasible allocations in a marginal, but important, way (the line segment between points $\mathbf{b}$ and $\mathbf{c}$ in Figure 4). That welfare may improve with the imposition of an additional constraint is consistent with what is known from the theory of the second-best.

\subsection{Related Literature}

The idea that information might be detrimental to economic welfare goes back at least to Hirshleifer (1971), who provides an example in which new information destroys an ex ante desirable risk-sharing arrangement. A great number of papers since then-primarily in the agency literature-have studied related phenomena; see, for example, Prat (2005). Citanna and Villanacci (2000) provide an early example of the phenomenon in the context of a dynamic general equilibrium model, but do not address the question of optimal disclosure.

Andolfatto (2010) uses the dynamic framework introduced by Lagos and Wright (2005) to investigate how information with zero social value can lead 
to welfare-reducing asset price movements when the asset in question is used to facilitate exchange. The environment we study here shares elements of the basic framework of Andolfatto (2010), but extends it in an important way. First, individuals in our set-up possess a costly information acquisition technology. It is the existence of this technology that gives rise to the threat of undue diligence in our model, and is what yields the trade-off that is absent in Andolfatto (2010). Second, we use a mechanism design approach to characterize the incentivefeasible allocations and the optimal communication policy, while Andolfatto (2010) studies competitive equilibria.

Morris and Shin (2002) are interested in the issue of optimal information disclosure. These authors investigate the properties of a generic model of strategic complementarity. Public information conveys information on underlying fundamentals (as it does in our model), but it also serves as a focal point for individual beliefs (unlike our model). Owing to the "information externality" that is present in their environment, individuals may "overreact" to public information if it is disclosed too precisely. So as with our model, society is faced with a trade-off in terms of disclosure policy.

It is interesting to compare a few key results from Morris and Shin (2002) with properties of our model. First, in their set-up, a greater provision of public information always increases welfare when individuals have no socially valuable private information. Note that individuals in our model have no socially valuable private information; and yet, our trade-off remains. Second, the trade-off becomes relevant in their setting only when individuals have access to statistically independent sources of information. Again, individuals in our model have no statistically independent information (although, they have independent means of learning the same information); and yet, our trade-off remains. It seems apparent then that the trade-off arises in our model for completely unrelated reasons.

A paper closely related to our analysis is Dang, Gorton, and Holmström (2010); henceforth, DGH. ${ }^{16}$ These authors examine a simple stage game and explore the question of optimal security design when agents have an information acquisition technology similar to the one in our model. They demonstrate that a debt contract is the least information-sensitive security, because it minimizes the production of private information. ${ }^{17}$ There is a public signal that reveals information about the security's payoff after a primary market closes, but before a secondary market opens. If the news is bad, the information sensitivity of the security increases, its price consequently falls, and trading is reduced. According to the authors, the latter result corresponds to their definition of a systemic event; i.e., when "information-insensitive debt [becomes] information-sensitive, giving rise to concerns of adverse selection, [which] reduces the amount of trade below what could be implemented if the agents just traded at the lower expected

\footnotetext{
${ }^{16}$ See also Gorton and Ordoñez (2011).

${ }^{17} \mathrm{As}$ in our model, the use of the private inspection technology is suboptimal from a society's point of view.
} 
value of the debt." (see DGH, p. 3).

In contrast to DGH, we do not study the optimal security design, nor do we formally analyze systemic events such as a sudden break-down in trading triggered by public information (although we give some thoughts about the latter in our discussion section). Our goal is more modest. We take the payoffproperty of the security as exogenous, and ask the question how a particular information flow should be managed in different environments. Our mechanism design approach has the benefit of making clear that our conclusions follow from the properties of the environment, as opposed to being possibly artifacts of assumptions relating (say) to the choice of a trading protocol. In DGH, for example, the terms of trade are assumed to be determined by take-it-or-leave-it offers by the buyer of the asset in the first bilateral meeting and then by the seller of the asset in the second bilateral meeting. In each case, the uniformed agent makes the take-it-or-leave-it offer. This avoids complications that arise in games with asymmetric information (see e.g., Berentsen and Rocheteau 2005). Our mechanism approach avoids these complications, since agents are always either equally ignorant about the payoff of the security or symmetrically informed about it.

\section{Discussion}

We believe that the question studied here is related to the issue of what properties are desirable in exchange media, including monetary instruments and assets that are used as collateral in short-term lending arrangements (like the overnight repo market). One desirable property of a monetary instrument is that it circulates easily; that is, without too much attention paid to its fundamental attributes. A risk-free asset, if it were to exist, would serve this function well.

But the risk properties of an asset alone are not responsible for determining liquidity. Gorton (2009a) suggests that the liquidity of an asset depends on it being "informationally insensitive." That is, what seems to be important is that potential transactors are symmetrically informed (or uniformed). This property quite likely explains the liquidity value of U.S. dollars and U.S. treasury debt, for example. It may also explain the liquidity properties of certain private-label assets that were used extensively in the U.S. repo market prior to its collapse in $2008 .{ }^{18}$ If the sheer complexity of these products had one redeeming attribute, it virtually ensured that everyone was symmetrically ignorant of their underlying properties. And our model provides an example of how symmetric ignorance can enhance the liquidity property of an asset.

As is well-known, the financial crisis in 2008 was centered squarely in the U.S. repo market. Assets that had, a short time earlier, served easily as collateral were suddenly discounted very heavily. According to Gorton (2009a), assets

\footnotetext{
${ }^{18}$ For example, the AAA-rated tranches of mortgage-backed securities.
} 
that were previously thought to be informationally insensitive were no longer so. We think that our framework may be used to formalize this idea and provide interpretations of what may have transpired during that period.

\subsection{The Gorton hypothesis}

Gorton (2009a) argues that the financial crisis was essentially a bank run in the shadow banking sector, and that historically, bank runs take place when "informationally-insensitive" debt suddenly becomes "informationally sensitive" owing to some shock. According to Gorton, the shock in the recent crisis took the form of a "surprise" drop in real estate prices which, in turn, led to a shock in subprime mortgage values. Evidently, this shock interacted with a contemporaneous innovation in information dissemination. In 2006, a financial information services company called Markit Group launched a product called the ABX.HE index. This index is a credit derivative containing twenty equally-weighted subprime-backed, short-term bonds, each of different risk-return ratings. The product allowed people to price and trade subprime risk (and also, to short the subprime market).

Gorton (2009b) suggests that the market price of the ABX.HE index aggregated and communicated market views on mortgage-backed securities (MBS); views that prior to this were largely unknown. ${ }^{19}$ One way to interpret this technological innovation through the lens of our model is as an exogenous decline in $\gamma$. In particular, think of moving from region B to D in Figure 3. Individuals now have the incentive to inspect assets that are offered as payment or collateral. No serious repercussions are associated with this event, as long as the news remains good (e.g., real estate prices continue to rise). But if the event is associated with bad news (real estate prices that flatline, or begin to decline), then a severe financial crisis ensues. Asset prices plummet, debt constraints bind, and output contracts. This is similar to the systemic event described in DHG.

\subsection{The Isaac hypothesis}

Former FDIC chair William Isaac, among many other commentators, suggest that the financial crisis in 2008 was exacerbated by the "transparency law" FASB rule 157. As it turns out, this "mark-to-market" accounting legislation was introduced in 2006 (implemented in 2007)-precisely at the time that U.S. real estate prices began to weaken. The subsequent drop in real estate prices, together with FASB 157, evidently forced banks to record large losses for the MBS assets they owned. This, combined with regulatory stipulations governing

\footnotetext{
${ }^{19}$ According to Gorton (2009b, p. 567), the ABX.HE indices "were the only place where a subprime related instrument traded in a transparent way, aggregating and revealing information about subprime residential mortgage-backed securities."
} 
capital ratios compelled banks to contract their lending activities, dispose of assets, and hoard cash.

In the context of our model, we might interpret this event as the economy being at a point in region $\mathrm{B}$ or $\mathrm{C}$, where efficiency dictates nondisclosure. We might then interpret FASB 157 as an exogenous (inefficient) policy imposition that, combined with bad news, leads to a financial crisis.

\subsection{An increase in macroeconomic uncertainty}

We can say a few words here about a change in uncertainty, modeled here as a mean-preserving increase of the spread $z_{h}-z_{l}$. From (13), a mean-preserving increase of $z_{h}-z_{l}$ decreases the curve labeled $\bar{\gamma}$ in Figure 3 (see footnote 15). From equations (15) and (23), it increases the curves labelled $\bar{z}^{F}$ and $\bar{z}^{D}$, respectively. Note that the curve labelled $\bar{z}^{N}$ is not affected. Figure 5 indicates how the regions of Figure 3 are modified. The regions for the initial spread are characterized by the dotted curves and the regions for the final spread are characterized by the solid curves.

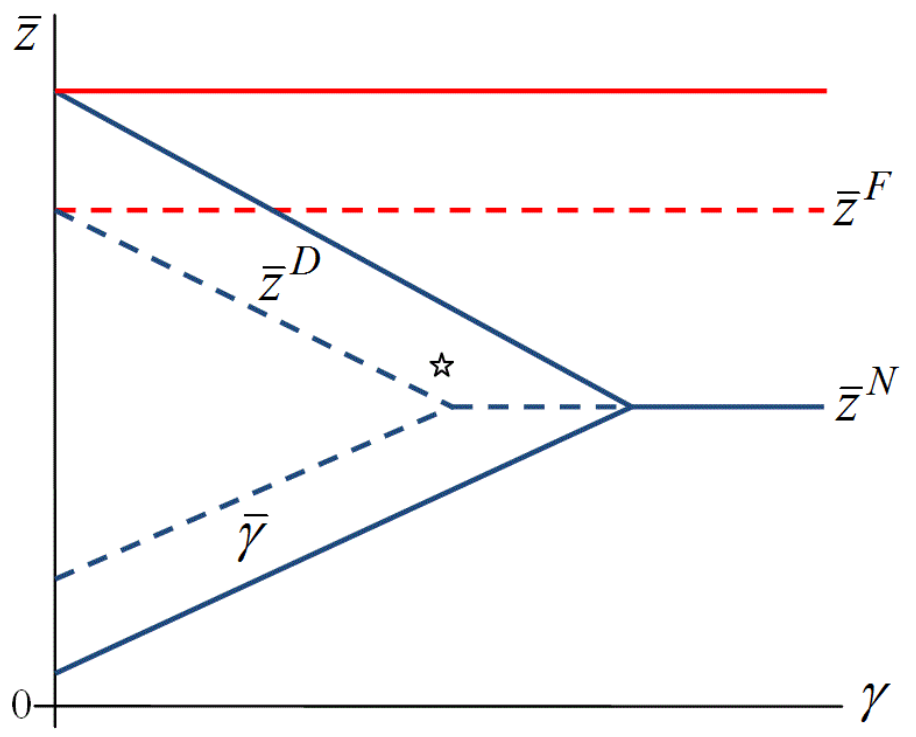

Figure 5: Mean preserving increase in the spread.

Suppose the economy is characterized by the pair $\star=(\gamma, \bar{z})$ which is indicated by the star in Figure 5. Suppose further that the initial spread is $d^{0}$ which then over time increases to $d^{1}$. For the initial spread $d^{0}$, the economy is in region B where the efficient night allocation $\left(q^{*}, q^{*}\right)$ is produced. As the spread starts to increase, the economy will continue to produce $\left(q^{*}, q^{*}\right)$ as long as the 
spread is not too large. ${ }^{20}$ However, once the spread crosses a certain treshhold, the economy will enter region $\mathrm{D}$. In region $\mathrm{D}$, the no-inspection condition is binding and night output starts declining. ${ }^{21}$ Note that initially no crisis occurs, since the only effect is that night output starts to decline gradually. However, at some further threshold, it will become optimal to turn the communication switch on and to choose the state contingent allocation. If it happens to be the case, that when the switch is turned on the state is low, the economy faces a sudden drop in the night output; i.e., a crisis occurs.

\section{Conclusion}

When commitment is lacking, assets can possess value beyond their underlying income stream. In particular, they can be valued for their ability to facilitate intertemporal trade. When assets possess "liquidity value," it is possible that some types of information relating to their future asset return are better left hidden (or disclosed with delay). "Bad news" events that lead to temporary downward revisions in asset returns depress asset values. This is perfectly normal and largely inconsequential (from a policy perspective) in frictionless settings. However, when commitment is lacking, there is the added bad effect of tightening debt constraints-leading to a "credit crunch." ${ }^{22}$ This is one reason, among several others identified in the literature, for why a full disclosure policy is not necessarily the best disclosure policy.

On the other hand, even information that is ideally left hidden should be disclosed if individuals have the ability to discover it for themselves at low cost. This corresponds to rendering exchange media in decentralized settings perfectly transparent. The result is the occasional credit crunch, but nevertheless constitutes the constrained efficient solution. Because of undue diligence (and possibly other factors identified in the literature), society may face a trade-off in designing policies that either promote or discourage information disclosure.

\footnotetext{
${ }^{20}$ Note that only the regions in Figure 5 expand, since the economy $\star=(\gamma, \bar{z})$ remains the same.

${ }^{21}$ This can be also seen from Figure 2, where a mean-preserving increase in the spread shifts the curve labeled $\beta^{-1} h(q)+(\beta \Omega)^{-1}(\bar{\gamma}-\gamma)$ to the left.

${ }^{22}$ In our model, tightening debt constraints are reflected in the mechanism's inability to offer a high enough future reward to induce sequential participation at the first-best allocation.
} 


\section{Appendix}

Proof of Proposition 1. To begin, we assign zero surplus to the buyer; i.e., $y_{b}^{i}+\bar{u}=0, i=l, h$. In this manner, the seller can be allocated the maximum incentive to produce output $\left(q^{l}, q^{h}\right)$ at night. Consequently, we set (9) to equality in what follows. The resource constraints (3) then imply $y_{s}^{l}=z^{l}+\bar{u}$ and $y_{s}^{h}=z^{h}+\bar{u}$. We can use these latter expressions to write the seller SP constraints as follows:

$$
\begin{aligned}
-h\left(q^{l}\right)+\beta\left(\bar{u}+z^{l}\right) & \geq\left(\beta^{T}-\beta\right) V_{s} \\
-h\left(q^{h}\right)+\beta\left(\bar{u}+z^{h}\right) & \geq\left(\beta^{T}-\beta\right) V_{s} .
\end{aligned}
$$

We then use (25) and (26) to define the sets (10) and (11). Given the assumed properties for $h(q)$ and $u(q)$, the sets $S^{l}$ and $S^{h}$ are non-empty and strictly convex. The set of incentive-feasible quantities $\left(q^{l}, q^{h}\right)$ is given by $S^{l} \cap S^{h}$; which is a non-empty and compact set.

Proof of Proposition 2. If we set $q^{l}=q^{h}=q^{*}$ in (25) and rearrange the resulting expression, we get $\bar{z} \geq \bar{z}^{F}$.

Proof of Proposition 3. Conditions (17) and (19) imply that any incentivefeasible allocation must satisfy

$$
\beta^{-1} h(q) \leq \bar{y}_{s} \leq \bar{z}+u(q) .
$$

Define $\Psi(q) \equiv \beta[\bar{z}+u(q)]-h(q)$. The function $\Psi(q)$ is strictly concave with $\Psi(0)=\beta \bar{z}$. Furthermore, there is a unique maximizer $\hat{q}$ that solves $\beta u^{\prime}(\hat{q})-$ $h^{\prime}(\hat{q})=0$. It follows that $\Psi(q)$ is increasing in $q \in(0, \hat{q})$ and decreasing for $q>\hat{q}$. For $q_{m}$, the incentive-feasible allocation is unique and satisfies $\left(q, \bar{y}_{s}\right)=$ $\left(q_{m}, \bar{z}+u\left(q_{m}\right)\right)$. For any $0 \leq q<q_{m}$, condition (27) implies $h(q)<\beta[\bar{z}+u(q)]$ and so there exists a continuum of incentive-feasible allocations $\left(q, \bar{y}_{s}\right)$ with $\bar{y}_{s} \in\left[\beta^{-1} h(q), \bar{z}+u(q)\right]$. Note that the expected transfer $\bar{y}_{s}$ only affects how the surplus is shared.

Proof of Proposition 4. Condition (27) suggests that we can choose parameters $\beta$ and $\bar{z}$ so that the following condition holds:

$$
\beta^{-1} h\left(q^{*}\right)=\bar{y}_{s}^{*}=\bar{z}+u\left(q^{*}\right) .
$$

That is, condition (28) identifies parameters such that the efficient allocation is just incentive feasible; i.e., it leaves both seller and buyer on their respective SP constraints. It is then clear that $q^{*}$ is incentive feasible if $\bar{z} \geq \bar{z}^{N}$.

Proof of Proposition 5. Recall that the seller's SP constraint is $\bar{y}_{s} \geq$ $\beta^{-1} h(q)$. Hence, if $\gamma<\bar{\gamma}$, then (22) implies that $N \subset S$; which immediately implies that $N \cap B \subset S \cap B$.

Proof of Proposition 6. Conditions (19) and (22) imply that any incentivefeasible allocation that does not promote inspection by the seller must satisfy

$$
\beta^{-1} h(q)+(\beta \Omega)^{-1}(\bar{\gamma}-\gamma) \leq \bar{y}_{s} \leq \bar{z}+u(q) .
$$


Define

$$
\Psi_{0}(q) \equiv \bar{z}+u(q)-\beta^{-1} h(q)-(\beta \Omega)^{-1}(\bar{\gamma}-\gamma) .
$$

The function $\Psi_{0}(q)$ is strictly concave. Furthermore, $\Psi_{0}(q)$ has an unique maximum at $\hat{q} \in\left(0, q_{0}\right)$ satisfying $\beta u^{\prime}(\hat{q})-h^{\prime}(\hat{q})=0$. Therefore $\Psi_{0}(q)$ is increasing over $q<\hat{q}$ and decreasing over $q>\hat{q}$. At $q_{0}$, the incentive-feasible allocation that does not promote information acquisition by the seller is unique and satisfies $\left(q, \bar{y}_{s}\right)=\left[q_{0}, \bar{z}+u\left(q_{0}\right)\right]$. For any $0 \leq q<q_{0}, \beta^{-1} h(q)<\bar{z}+u(q)$; there exists a continuum of incentive-feasible allocations that do not promote inspection by the seller and that differ only in the expected transfer $\bar{y}_{s}$.

Proof of Proposition 7. Condition (29) suggests that we can choose parameters $\beta$ and $\bar{z}$ so that the following condition holds:

$$
\beta^{-1} h\left(q^{*}\right)+(\beta \Omega)^{-1}(\bar{\gamma}-\gamma)=\bar{y}_{s}^{*}=\bar{z}+u\left(q^{*}\right)
$$

That is, condition (30) identifies parameters such that the efficient allocation is just sufficient to discourage information acquisition by the seller. It is then clear that $q^{*}$ does not promote inspection by the seller if $\bar{z} \geq \bar{z}^{D}$.

Furthermore, the seller's participation constraint must also not bind; i.e., $\bar{z} \geq$ $\bar{z}^{N}$. It then follows that the efficient night output $q^{*}$ requires that $\bar{z} \geq \max \left\{\bar{z}^{N}, \bar{z}^{D}\right\}$. Note that at $\gamma=\bar{\gamma}, \bar{z}^{N}=\bar{z}^{D}$.

Proof of Proposition 8. If the switch is turned off, $\bar{z} \leq \bar{z}^{N}$ and $\gamma \geq \bar{\gamma}$, then welfare of the constrained-efficient allocation satisfies

$$
(1-\beta) W\left(q_{m}, q_{m}\right)=u\left(q_{m}\right)-h\left(q_{m}\right)+\bar{z},
$$

where, from Proposition (3), $q_{m}$ satisfies

$$
\beta\left[\bar{z}+u\left(q_{m}\right)\right]-h\left(q_{m}\right)=0 .
$$

If the switch is turned on, denote $\left(q_{*}^{l}, q_{*}^{h}\right)$ the constrained-efficient night output. Since the set $S^{l} \cap S^{h}$ is non-empty and compact, we know that such a $\left(q_{*}^{l}, q_{*}^{h}\right)$ exists. ${ }^{23}$ Welfare in this case satisfies

$$
(1-\beta) W\left(q_{*}^{l}, q_{*}^{h}\right)=\bar{u}_{*}-\bar{h}_{*}+\bar{z},
$$

where $\bar{u}_{*} \equiv \pi u\left(q_{*}^{h}\right)+(1-\pi) u\left(q_{*}^{l}\right)$ and $\bar{h}_{*} \equiv \pi h\left(q_{*}^{h}\right)+(1-\pi) h\left(q_{*}^{l}\right)$.

From (25) and (26), $\left(q_{*}^{l}, q_{*}^{h}\right)$ satisfies

$$
-\bar{h}_{*}+\beta\left(\bar{u}_{*}+\bar{z}\right) \geq 0 \text {. }
$$

To see this, multiply both sides of (25) by $1-\pi$ and both sides of (26) by $\pi$. Then, add the two equations and rearrange to get

$$
-\bar{h}_{*}+\beta\left(\bar{u}_{*}+\bar{z}\right) \geq\left(\beta^{T}-\beta\right) V_{s}
$$

\footnotetext{
${ }^{23}$ The quantities $\left(q_{1}^{l}, q_{1}^{h}\right)$ are not always the welfare maximizing quantities when the communication switch is on (see Figure 1). They are always the welfare maximizing quantities when $T=1$. However, when $T>1$ one can have $q_{1}^{l}<q^{*}<q_{1}^{h}$. In this case, it can be optimal to reduce $q_{1}^{h}$ even when it might involve reducing $q_{1}^{l}$ too.
} 
Next, replace $V_{s}$ and rearrange to get

$$
\left[-\bar{h}_{*}+\beta\left(\bar{u}_{*}+\bar{z}\right)\right]\left(1-\beta^{T}\right)(1-\beta)^{-1} \geq 0 .
$$

Since $\left(1-\beta^{T}\right)(1-\beta)^{-1}>0$, condition (31) follows.

Consider first the case $q_{*}^{l} \neq q_{*}^{h}$. Then, concavity of $u(q)$ and $-h(q)$ imply that

$$
-h\left(\bar{q}_{*}\right)+\beta\left[u\left(\bar{q}_{*}\right)+\bar{z}\right]>-\bar{h}_{*}+\beta\left(\bar{u}_{*}+\bar{z}\right) \geq 0,
$$

where $\bar{q}_{*}=\pi q_{*}^{h}+(1-\pi) q_{*}^{l}$. The term on the left-hand side of the strict inequality is equal to zero at $\bar{q}_{*}=q_{m}$. Then, $-h^{\prime}\left(q_{m}\right)+\beta u^{\prime}\left(q_{m}\right)<0$ (see the proof of Proposition 3), implies $\bar{q}_{*}<q_{m}$.

Furthermore, welfare satisfies $u\left(\bar{q}_{*}\right)-h\left(\bar{q}_{*}\right)+\bar{z} \geq \bar{u}_{*}-\bar{h}_{*}+\bar{z}$, which again follows from the concavity of $u(q)$ and $-h(q)$. Then, since $\bar{q}_{*}<q_{m}$, we have

$$
u\left(q_{m}\right)-h\left(q_{m}\right)+\bar{z}>u\left(\bar{q}_{*}\right)-h\left(\bar{q}_{*}\right)+\bar{z} \geq \bar{u}_{*}-\bar{h}_{*}+\bar{z} .
$$

Thus, we have just demonstrated that if $q_{*}^{l} \neq q_{*}^{h}$, it is optimal to turn the communication switch off and set $q_{l}=q_{h}=q_{m}$.

Consider next the case $q_{*}^{l}=q_{*}^{h}=q_{*}$. If we set $q^{l}=q^{h}=q_{*}$ in (25) and rearrange the resulting expression, we get

$$
\beta=\frac{h\left(q_{*}\right)}{u\left(q_{*}\right)+\bar{z}}+\frac{\bar{\gamma}}{\Omega\left[u\left(q_{*}\right)+\bar{z}\right]} .
$$

The quantity $q_{m}$ satisfies $\beta\left[\bar{z}+u\left(q_{m}\right)\right]-h\left(q_{m}\right)=0$. Use this expression to replace $\beta$ on the left-hand side to get

$$
\frac{h\left(q_{m}\right)}{u\left(q_{m}\right)+\bar{z}}=\frac{h\left(q_{*}\right)}{u\left(q_{*}\right)+\bar{z}}+\frac{\bar{\gamma}}{\Omega\left[u\left(q_{*}\right)+\bar{z}\right]}
$$

which immediately implies that $q_{m}>q_{*}$. Thus, we have just demonstrated that if $q_{*}^{l}=q_{*}^{h}$, it is also optimal to turn the communication switch off and set $q_{l}=q_{h}=q_{m}$.

We thus conclude that if $\bar{z} \leq \bar{z}^{N}$ and $\gamma \geq \bar{\gamma}$, the optimal communication policy is to turn the switch off and to choose the allocation $\left(q, \bar{y}_{s}\right)=$ $\left(q_{m}, \bar{z}+u\left(q_{m}\right)\right)$.

Proof of Proposition 9. Let $\gamma \leq \bar{\gamma}$ and $\bar{z}<\bar{z}^{D}$. To prove Proposition 9, we do not need to know the exact details of the constrained-efficient allocation when the communication switch is on. Since the set $S^{l} \cap S^{h}$ is non-empty and compact, we know that such an allocation exists. Let $\left(q_{*}^{l}, q_{*}^{h}\right)$ denote such an allocation.

If the switch is on, welfare of the constrained-efficient allocation satisfies

$$
(1-\beta) W\left(q_{*}^{l}, q_{*}^{h}\right)=\bar{u}_{*}-\bar{h}_{*}+\bar{z}
$$


where $\bar{u}_{*} \equiv \pi u\left(q_{*}^{h}\right)+(1-\pi) u\left(q_{*}^{l}\right)$ and $\bar{h}_{*} \equiv \pi h\left(q_{*}^{h}\right)+(1-\pi) h\left(q_{*}^{l}\right)$. Note that since $\bar{z}<\bar{z}^{F}$, the efficient allocation is not incentive feasible, implying that $W\left(q_{*}^{l}, q_{*}^{h}\right)<W\left(q^{*}, q^{*}\right)$.

If the switch is off, welfare of the constrained-efficient allocation satisfies

$$
(1-\beta) W\left(q_{0}, q_{0}\right)=u\left(q_{0}\right)-h\left(q_{0}\right)+\bar{z} .
$$

The remainder of the proof involves four steps. First we show that $W\left(q_{0}, q_{0}\right)$ is increasing in $\gamma$. Second, we evaluate $W\left(q_{0}, q_{0}\right)$ at $\gamma=0$ and show that $W\left(q_{*}^{l}, q_{*}^{h}\right)>W\left(q_{0}, q_{0} \mid \gamma=0\right)$. Third, we evaluate $W\left(q_{0}, q_{0}\right)$ at $\gamma=\bar{\gamma}$ and show that $W\left(q_{*}^{l}, q_{*}^{h}\right)<W\left(q_{0}, q_{0} \mid \gamma=\bar{\gamma}\right)$. Fourth, we conclude that there exists a $0<\tilde{\gamma}<\bar{\gamma}$ such that $W\left(q_{*}^{l}, q_{*}^{h}\right)=W\left(q_{0}, q_{0} \mid \gamma=\tilde{\gamma}\right)$.

First step. $W\left(q_{0}, q_{0}\right)$ is strictly increasing in $\gamma \leq \bar{\gamma}$. To see this, note that from (29) at equality we get

$$
\frac{d q_{0}}{d \gamma}=-\frac{1}{\left[\beta u^{\prime}\left(q_{0}\right)-h^{\prime}\left(q_{0}\right)\right] \Omega}>0
$$

since $\beta u^{\prime}\left(q_{0}\right)-h^{\prime}\left(q_{0}\right)<0$ (see the proof of Proposition 6 ), and so

$$
\frac{d W\left(q_{0}, q_{0}\right)}{d \gamma}=\left[u^{\prime}\left(q_{0}\right)-h^{\prime}\left(q_{0}\right)\right] \frac{d q_{0}}{d \gamma}>0
$$

since $q_{0}<q^{*}$ if $\gamma<\bar{\gamma}$.

Second step. Evaluate night output at $\gamma=0$. From (29), at $\gamma=0, q_{0}$ satisfies

$$
\beta^{-1} h\left(q_{0}\right)+(\beta \Omega)^{-1} \bar{\gamma}=\bar{z}+u\left(q_{0}\right)
$$

Next, consider (25) at equality. Setting $q^{l}=q^{h}=q$ and rearranging yields

$$
\beta^{-1} h(q)+(\beta \Omega)^{-1} \bar{\gamma}=\bar{z}+u(q)
$$

Thus, the quantities $q^{l}=q^{h}=q_{0}$ satisfy (25) at equality. This result clearly implies that $W\left(q_{*}^{l}, q_{*}^{h}\right)>W\left(q_{0}, q_{0} \mid \gamma=0\right)$ (this is point $c$ in Figure 3$)$.

Third step. Evaluate night output at $\gamma=\bar{\gamma}$. We distinguish the following two cases: $\bar{z} \geq \bar{z}^{N}$ and $\bar{z}<\bar{z}^{N}$.

Case $\bar{z} \geq \bar{z}^{N}$. From Proposition $7, \bar{z}^{D}=\bar{z}^{N}$ at $\gamma=\bar{\gamma}$. Hence, at $\gamma=\bar{\gamma}$ we have $q_{0}=q^{*}$. Thus, if the communication switch is off and if $\gamma=\bar{\gamma}$, we have $q_{0}=q^{*}$. Thus, in this case $W\left(q_{*}^{l}, q_{*}^{h}\right)<W\left(q_{0}, q_{0} \mid \gamma=\bar{\gamma}\right)=W\left(q^{*}, q^{*}\right)$.

Case $\bar{z}<\bar{z}^{N}$. From Proposition $7, \bar{z}^{D}=\bar{z}^{N}$ at $\gamma=\bar{\gamma}$. Hence, at $\gamma=\bar{\gamma}$ we have $q_{0}=q_{m}$. In this case, from Proposition 8 , we also have $W\left(q_{*}^{l}, q_{*}^{h}\right)<$ $W\left(q_{0}, q_{0} \mid \gamma=\bar{\gamma}\right)$.

Fourth step. From the second and third steps we have that at $\gamma=0$ $W\left(q_{*}^{l}, q_{*}^{h}\right)>W\left(q_{0}, q_{0} \mid \gamma=0\right)$ and at $\gamma=\bar{\gamma} W\left(q_{*}^{l}, q_{*}^{h}\right)<W\left(q_{0}, q_{0} \mid \gamma=\bar{\gamma}\right)$. From the first step, $W\left(q_{0}, q_{0}\right)$ is monotonically increasing in $\gamma$. Recall that $W\left(q_{*}^{l}, q_{*}^{h}\right)$ is independent of $\gamma$. Hence, there exists a unique $\hat{\gamma} \in(0, \bar{\gamma})$ such that $W\left(q_{0}, q_{0}\right)=W\left(q_{*}^{l}, q_{*}^{h}\right)$. 


\section{References}

[1] Andolfatto, David (2010). On the Social Cost of Transparency in Monetary Economies," Federal Reserve Bank of St. Louis, working paper.

[2] Berentsen, Aleksander and Guillaume Rocheteau (2004). Money and Information. Review of Economic Studies, 71, 915-944.

[3] Citanna, Alessandro and Antonio Villanacci (2000). Incomplete Markets, Allocative Efficiency, and the Information Revealed by Prices. Journal of Economic Theory, 90, 222-253.

[4] Gorton, Gary and Guillermo Ordoñez (2011). Collateral Crises, manuscript.

[5] Lagos, Ricardo and Guillaume Rocheteau (2008). Money and capital as competing media of exchange, Journal of Economic Theory, 142, 247-258.

[6] Dang, Tri, Gorton, Gary and Bengt Holmström (2010). Financial Crises and the Optimality of Debt for Liquidity Provision. Yale University, manuscript, May 10, 2010.

[7] Ferraris, Leo and M. Watanabe (2008). Collateral secured loans in a monetary economy, Journal of Economic Theory, 143, 405-424.

[8] Gorton, Gary (2008). The Subprime Panic (September 30, 2008). Yale ICF Working Paper No. 08-25.

[9] Gorton, Gary (2009a). Slapped in the Face by the Invisible Hand: Banking and the Panic of 2007. Manuscript prepared for the Federal Reserve Bank of Atlanta's 2009 Financial Markets Conference: Financial Innovation and Crisis, May 11-13, 2009.

[10] Gorton, Gary (2009b). Information, Liquidity, and the (Ongoing) Panic of 2007. American Economic Review: Papers \& Proceedings 2009, 99:2, $567-572$.

[11] Lagos, Ricardo and Randall Wright (2005). A Unified Framework for Monetary Theory and Policy Analysis, Journal of Political Economy, 113, 463484 .

[12] Morris, Stephen and Hyun Song Shin (2002). Social Value of Public Information. American Economic Review, 92(5), 1521-1534.

[13] Prat, Andrea (2005). The Wrong Kind of Transparency. American Economic Review, 95(3), 862-877.

[14] Townsend, R.M., (1979). Optimal Contracts and Competitive Markets with Costly State Verification. Journal of Economic Theory, 22, 265-293. 